\title{
Flow Field Analysis and Performance Assessment Inside a Vaned Diffuser of a Laboratory-Type Centrifugal Pump
}

\author{
Abdelmadjid Atif* and Sara Sami
}

Laboratory of Energetic Mechanics and Conversion Systems, Faculty of Mechanical and Process Engineering, University of Science and Technology USTHB, Algiers, Algeria

\section{ARTICLE INFO}

Keywords:

Centrifugal Pump

Pump Diffuser

Vanned Diffuser

Pressure Recovery

Diffuser Effectiveness

\begin{abstract}
The paper refers to the analysis of flow fields inside a vaned diffuser and performance assessment of a laboratory-type centrifugal pump operating with air. The study deals with numerical simulation of the flow at design flow rate, with focus on velocity and pressure distributions across a diffuser passage. The aim is to highlight the flow structure how it leaves the impeller and evolves through the diffuser to understand the mechanism of pressure recovery. The performance assessment consists of evaluating diffuser effectiveness. The numerical results are compared to experimental measurements for validation.
\end{abstract}

\section{Introduction}

Diffusers are essential parts to convert efficiently by area expansion the high kinetic energy leaving the impeller of a centrifugal pump into needed static pressure, what is called pressure recovery. The flow at entry of diffusers is extremely distorted, consisting of unsteady jet and wake structures issuing from each impeller passages, contributing into the complexity of the flow pattern throughout diffusers [(Dou \& Misuki, 1998), (Krain, 1981)]. All analysis procedures should take into account these flow conditions to assess the pressure recovery and performance.

A wide variety of diffuser designs are applied for this purpose (Japikse, 1984). The vaneless diffuser is often adopted as principal mean of pressure recovery owing to its simplicity, inexpensive construction and broad operating range. However, the long flow path due to inlet swirl angle and required space for diffusion, results in high friction loss [(Stanitz, 1952), (Johnston \& Dean, 1966)]. The commonly adopted approach to shorten the flow path is to insert guide vanes inside diffuser, in either cascade or channel configuration. In addition to giving improved performance, vaned diffusers provide the ability to reduce the overall size. The main disadvantage is reduced efficiency at off-design flow conditions, due to incidence effects on the vane leading edge, and therefore a reduced operating range [(Runstadler \& Dolan, 1973), (Dawes, 1995), (Bayeul-laine et al., 2016)].

\section{Experimental Procedure}

Tests have been performed in a laboratory-type impeller operating with air for convenience, on a test rig specially adapted to study impeller-diffuser flows (Fig. 1). The flow can be analyzed both with vaneless and vaned diffuser configurations. The flow at the outlet of diffuser discharges directly into surrounding atmosphere. The diffuser consists of two parallel flat disks, in which the vanes of specific geometry bulky blades fill up the inter-space between hub and shroud, and make up the diffuser passages (Fig. 2).

* Corresponding Author E-Mail Address: aatif@usthb.dz 


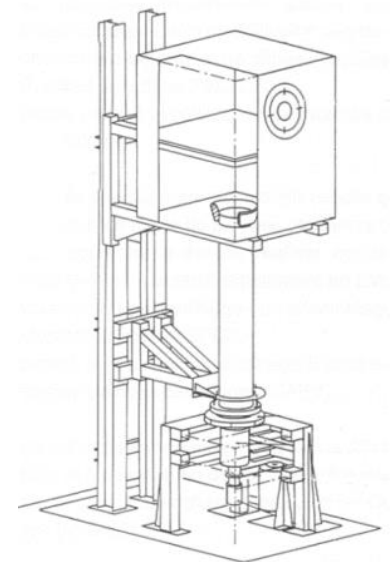

Figure 1. Experimental rig

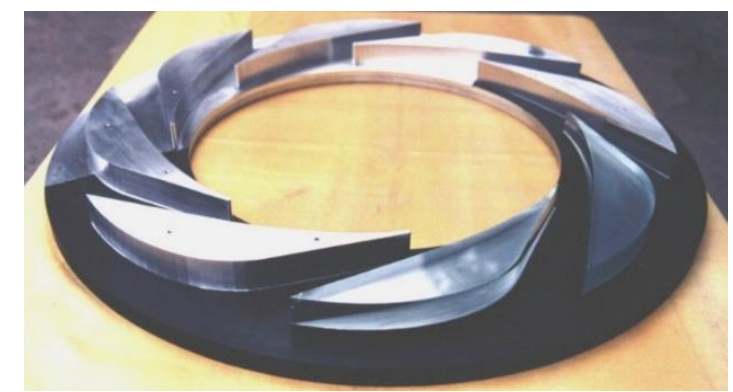

Figure 2. Diffuser vanes

The experimental investigations are conducted inside diffuser passages by a tiny 3-hole pressure probe (Fig. 3). The probe size is assumed to be small enough to not disturb the flow pattern. The probe, with prior and proper calibration, allows for the determination of static pressure, total pressure, and velocity and flow angle. All measurements are time-averaged.
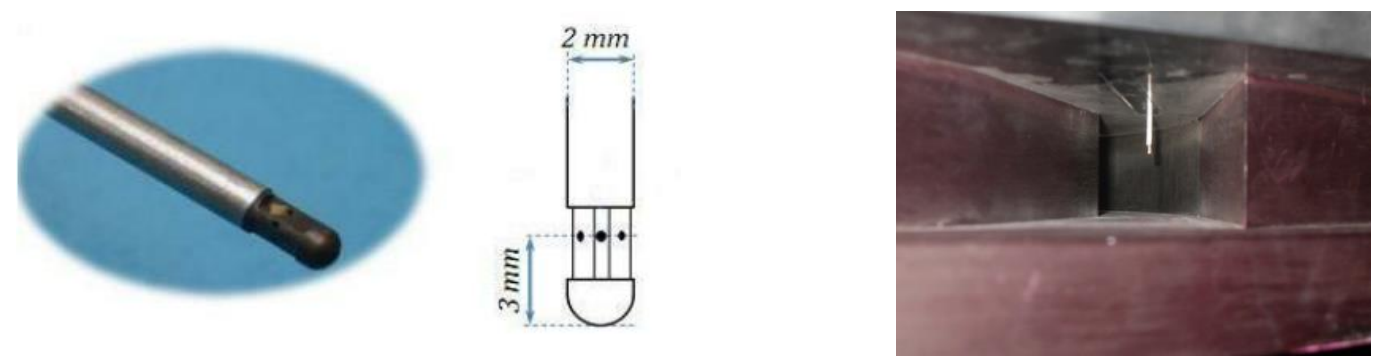

Figure 3. Probe exploration

Explorations are made at locations distributed all over the entry line, exit line and along the mid-line of a diffuser passage (Fig. 4), with traverse locations from hub to shroud at each line location. Further details can be found in (Cherdieu et al., 2013).

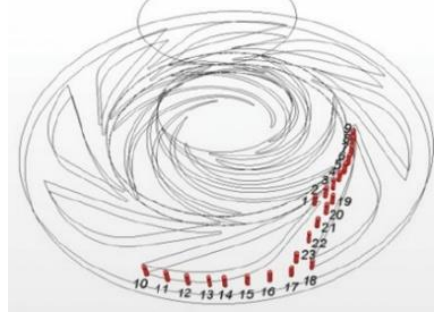

Figure 4. Measurement locations

\section{Numerical Procedure}

\section{3-1. Introduction}

The flow analysis is carried out for the entire pump, in which the diffuser is part of it. The purpose is to account for the interaction effects between the impeller and the diffuser vanes that might influence the flow structure (Bayeul-laine et al., 2016). Among all the results, the 
study mainly focuses on the flow pattern inside a vaned diffuser passage, to assess the pressure recovery and performance.

\section{3-2. Pump Modeling}

The pump is modeled as a set of three components: an entry duct, impeller and vaned diffuser (Fig. 5). Impeller rotates clockwise. The entry duct is chosen long enough to assume uniform upstream inlet flow conditions.

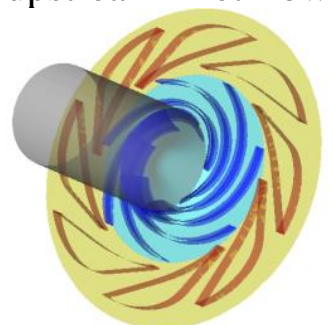

Figure 5. Pump geometry

\section{3-3. Test Conditions}

The numerical simulation of the complete $360^{\circ}$ impeller and diffuser is carried out for a steady flow approach with the so-called Frozen Rotor, in order to characterize part of the unsteady effects that appear within the impeller when it rotates in front of the stationary vanned diffuser [(Majidi, 2005), (Meakhail \& Park, 2005)].

The pump is tested at the same conditions as experimental procedure, at a flow rate close to diffuser design $\left(Q^{*}=0.771\right)$. It should be noted that the diffuser is designed to be fitted at $80 \%$ of the impeller design flow rate. This is intended to improve performance at low flow rates. The main features of the pump components and specification of operating conditions are summarized in Tab. 1.

Table 1.

Specifications of the pump

\begin{tabular}{lr}
\hline Test conditions & $1710 \mathrm{rpm}$ \\
\hline Speed of rotation & Air \\
Normalized flow rate $Q^{*}=Q / Q_{n}=0.771$ \\
Fluid
\end{tabular}




\section{3-4. Mesh Structure}

The pump is completely meshed with unstructured tetras, with prismatic layers attached to walls to account for boundary layers growth. Tetras are flexible elements to model complex passage geometries but the outcome is a larger problem dimension. Mesh refinements are placed at particular locations like blade edges, walls and components interface (Fig. 6). Mesh characteristics for each of the three components of the pump are listed in Tab. 2.

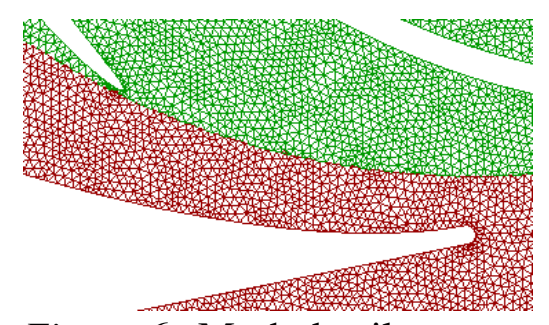

Figure 6. Mesh details

Table 2.

Mesh information

\begin{tabular}{lrc}
\hline Component & Elements & Nodes \\
\hline Entry duct & 660047 & 151735 \\
Impeller & 2835353 & 582772 \\
Diffuser & 696599 & 180538 \\
Total & 4191999 & 915045 \\
\hline
\end{tabular}

\section{3-5. Computations}

Computations are performed using licensed CFX CFD code. Three-dimensional, steady incompressible Reynolds averaged Navier-Stokes are solved, in combination with specific wall and interface models treatment as recommended for turbomachinery flows. The turbulence is modeled with $\mathrm{k}-\varepsilon$. The main features are summarized in Tab. 3.

\section{Table 3.}

Computation details

\begin{tabular}{ll} 
Inlet section of entry duct & Specified mass flow rate \\
Outlet section of diffuser & Constant zero pressure [Referred to atmospheric pressure] \\
Walls & Smooth and no slip \\
Fluid & Constant property Air at $25^{\circ} \mathrm{C}$ \\
Turbulence model & $\mathrm{k}-\varepsilon$ \\
Maximum number of iterations & 200 \\
Convergence criteria & Mass residue less than $10^{-5}$ \\
\hline
\end{tabular}

\section{3-6. Post-Process}

Simulation data are extracted on a plane located mid-span between the hub and shroud of the diffuser. This allows illustrating velocity, static and total pressure fields at the diffuser midspan. Furthermore, numerical data are mass flow-averaged at pressure probe measurement locations at mid-span of the diffuser to allow for comparison with experimental results.

\section{Results and Discussion}

\subsection{Velocity}

Figure 7 shows the distribution of velocity $\boldsymbol{V}$ in the mid-span plane of a vaned diffuser passage, in both vector and magnitude plots. The figure shows clearly the distorted flow as it leaves the 
impeller. This comes from the jet (high velocity) and wake (low velocity) flow structure inside the rotating passages of the impeller.

In the semi-vaned space, the flow is re-structuring before entry to the diffuser passage throat. Across the passage, it is slowing down more quickly at vane pressure side than suction side, and more at the vane sides than in the mid-line.

The vector plot shows good turning of the flow around the vane leading edge and across the passage. This is expected since the simulation is carried out at a flow rate very close to diffuser design one, and indicates that the flow direction fits well the geometry of the diffuser.

Far downstream in the diffuser passage, we notice a slight blockage effect due to boundary layer separation on both sides of the vanes. A wake is arising behind the trailing edge of the diffuser vanes.

At the exit of the vaned space, flows from different diffuser passages are gathering out and leaves the diffuser outlet in a rather distorted pattern.
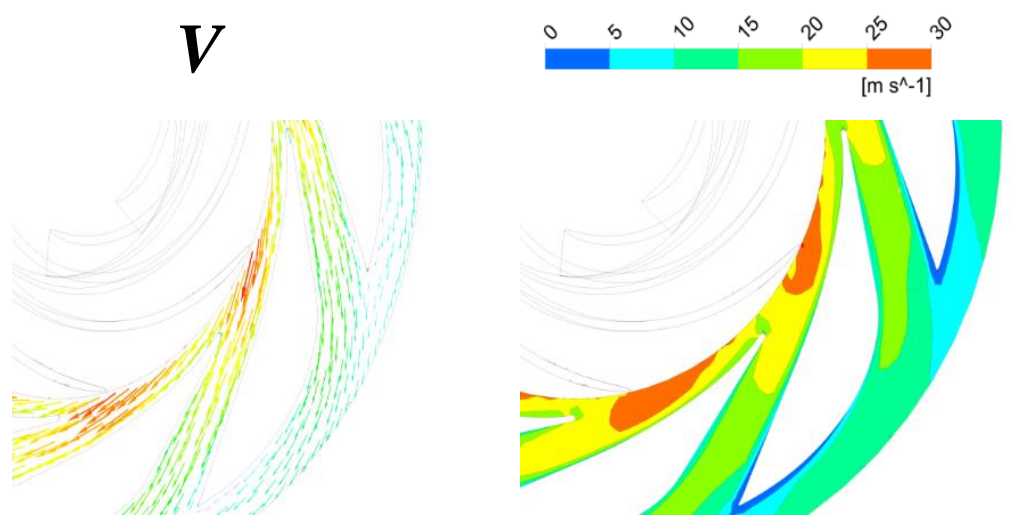

Figure 7. Velocity distribution

\subsection{Static and Total Pressure}

Figure 8 shows the distributions of respectively static pressure $\boldsymbol{P}$ and total pressure $\boldsymbol{P}_{\boldsymbol{t}}$ in the mid-span plane of a vaned diffuser passage.

For the static pressure, away from the area around the trailing edge of the impeller blades and the leading edge of the diffuser vanes, the figure shows a static pressure regularly and uniformly increasing across the diffuser passage up to the outlet gage atmospheric pressure. Small areas of under-pressure and over-pressure are observed at the exit of the vaned space on the suction side and downstream from the trailing edge respectively. The latter indicates a wake zone.

For the total pressure, the figure shows a decreasing total pressure across the diffuser passage due to friction and shocks, up to the gage outlet total pressure. Most of the total pressure loss is occurring at vane walls due to boundary layer growth, much more on the suction side than the pressure side. Total pressure loss is also substantial downstream from the trailing edge, because of separation and wake zone.
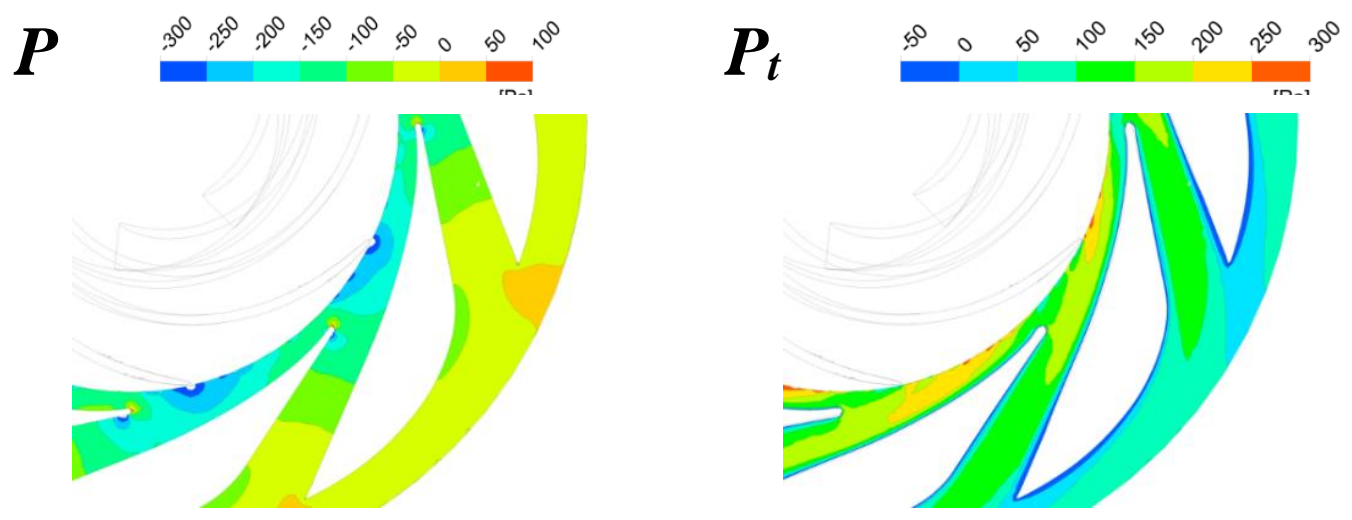
Figure 8. Static and total pressure distributions

\subsection{Comparison with experiment}

Figure 9 shows the experimental data together with the numerical curves for the static and total pressure respectively, at the mid-line and mid-span of the diffuser passage. The numerical results are mass flow-averaged over the circumferential section at the same location as the experimental one.

For the static pressure, the numerical and experimental profiles show a similar and a regularly increasing static pressure across the passage up to the gage atmospheric outlet pressure. However, a large discrepancy is observed in the inlet zone of the passage. The numerical simulation tends to over-estimate the static pressure.

For the total pressure, the numerical and experimental profiles show a similar and a regularly decreasing total pressure across the passage up to the gage outlet total pressure. However, a large discrepancy is observed in the inlet zone of the passage. The numerical simulation tends to under-estimate the total pressure.

Numerical-experimental discrepancies can be explained by several causes. First main cause is the fact that the numerical procedure is based on approximations: steady state assumption, geometry modeling, mesh refinement dependency, turbulence model, boundary conditions, and data processing. In addition, it must be pointed out that are some shortcomings of using pressure probe for measurements; sources of errors are calibration sensitivity, flow disturbances, unsteady effects, instruments uncertainties, and data processing.
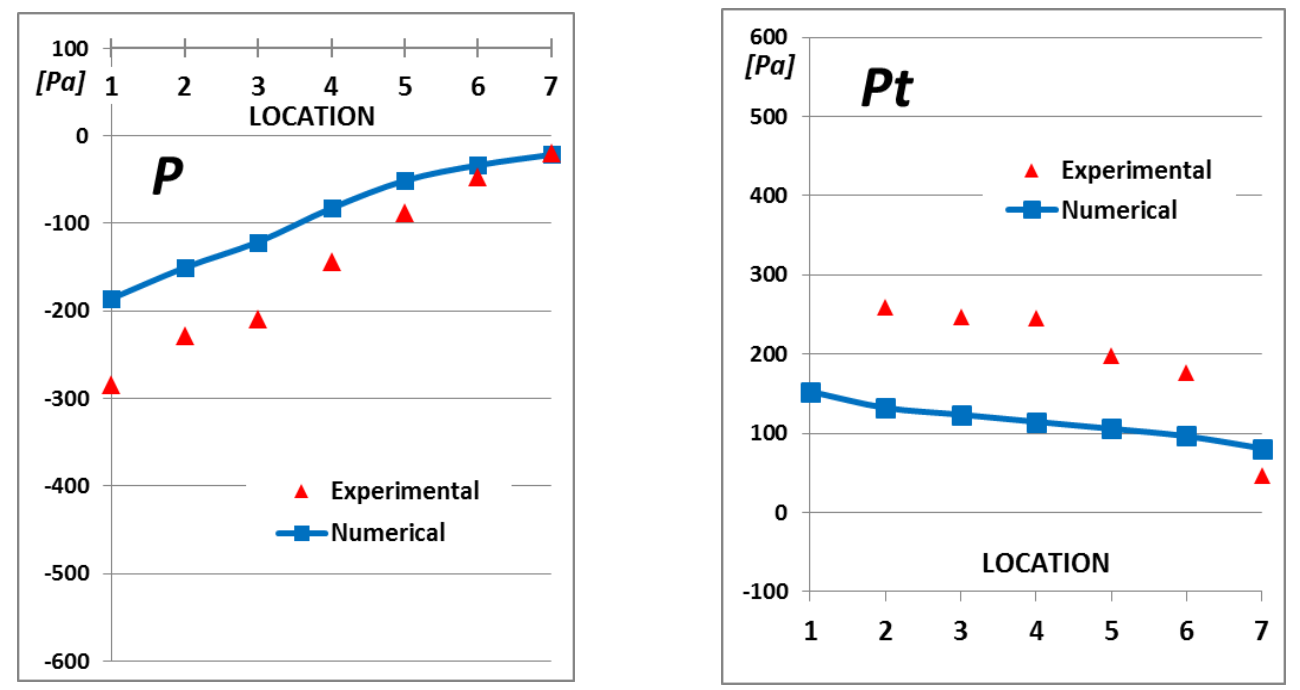

Figure 9. Numerical/Experimental comparison of static and total pressure plot

\subsection{Performance assessment}

The pressure recovery coefficient of a diffuser is defined as the ratio of the static pressure rise over the available velocity pressure at inlet:

$$
\boldsymbol{C}_{p}=\frac{\boldsymbol{P}_{\text {out }}-\boldsymbol{P}_{\text {in }}}{\boldsymbol{P}_{\text {tin }}-\boldsymbol{P}_{\text {in }}}
$$

The ideal pressure recovery coefficient is given by:

$$
C_{p i}=1-\frac{1}{A R^{2}}
$$

where $A R$ is the area ratio of outlet to inlet of the diffuser. That is $C_{p i}=0.66$ for the case studied. 
The diffuser effectiveness is defined as:

$$
\xi=\frac{C_{p}}{C_{p i}}
$$

Firstly, the pressure recovery coefficient is estimated from the computed static pressure rise and total pressure at inlet. The values are mass flow-averaged over the circumferential section at inlet and outlet of the diffuser. Secondly, the pressure recovery coefficient is calculated from the measured static and total pressures across the diffuser.

The diffuser performance is summarized in Tab. 4.

Table 4.

Performance Assessment

\begin{tabular}{ccc}
\hline & $\boldsymbol{C}_{\boldsymbol{p}}$ & $\boldsymbol{\xi}$ \\
\hline Simulation & 0.58 & 0.88 \\
Experiment & 0.56 & 0.85 \\
$\boldsymbol{\xi}$ for vaneless diffuser : & 0.5 \\
\hline
\end{tabular}

Both numerical and experimental values of $\boldsymbol{C}_{\boldsymbol{p}}$ and $\boldsymbol{\xi}$ are very close and give a high performance for the vaned diffuser, by contrast to a vaneless diffuser which has an effectiveness of about 0.5 .

It should be noted that this high performance is expected because both simulation and experiment have been conducted at vaned diffuser design flow rate. The performance should drop at off-design conditions.

\section{Conclusion}

A full pump impeller and diffuser flow steady numerical simulation has been carried out for the diffuser design flow rate. It focuses on the flow analysis inside a passage of the vaned diffuser. From analyzing such results, it appears clearly that the flow leaving the impeller is restructuring in the semi-vaned space to get into the diffuser passages. At this design flow rate, the flow direction fits well the diffuser geometry. Slight boundary layer separations are observed at the end of the vanes. A wake is arising behind the trailing edge of the vanes and the flow is leaving the diffuser outlet in a distorted pattern. The study shows a static pressure regularly and uniformly increasing across the diffuser passage with a decreasing total pressure. Most of the total pressure loss is occurring close to vane walls. For the static and total pressure, the numerical and experimental results show similar profiles but fairly the numerical simulation over-estimates the static pressure and under-estimates the total pressure. Finer numerical analysis is required to reduce these discrepancies. The pressure recovery coefficient and effectiveness are quite similar for both numerical and experimental studies and give a high performance for the vaned diffuser at its design flow rate. Further investigations should be carried out for off-design conditions.

\section{Acknowledgment}

Experimental measurements are gratefully provided by Patrick CHERDIEU from the Laboratoire de Mecanique de Lille, FRANCE.

\section{Nomenclature}

$$
\begin{array}{ll}
A R & \text { area ratio } \\
C_{p} & \text { pressure recovery coefficient }
\end{array}
$$




$\begin{array}{ll}C_{p i} & \text { ideal pressure recovery coefficient } \\ P & \text { static pressure }[\mathrm{Pa}] \\ P_{t} & \text { total pressure }[\mathrm{Pa}] \\ Q & \text { flow rate }\left[\mathrm{m}^{3} / \mathrm{s}\right] \\ V & \text { velocity }[\mathrm{m} / \mathrm{s}]\end{array}$

Greek

$\xi \quad$ effectiveness

Abbreviation

in inlet

out outlet

\section{References}

Bayeul-Laine, A.C., Dupont, P., Dazin, A. and Bois, G. (2016). Investigations inside a vaned diffuser of a centrifugal pump at low flow rates. Proceedings of the 28th Symposium on Hydraulic Machinery and Systems, Grenoble, France.

Cherdieu, P., Dazin, A., Dupont, P., Bayeul-Laine A.C., Roussette, O. and Bois, G. (2013). Analysis of unsteadinesses in a vaned diffuser of centrifugal machinery. Proceedings of the 23rd Congres Francais de Mecanique, Bordeau, France.

Dawes, W.N. (1995). Simulation of the Unsteady Interaction of a Centrifugal Impeller with its Vaned Diffuser: Flow Analysis. Journal of Turbomachinery, Vol. 117, pp. 213-222.

Dou, H. S. and Mizuki, S. (1998). Analysis of the Flow in Vaneless Diffusers with Large Width-to-Radius Ratios. Journal of Turbomachinery, Vol. 120, pp. 193-201.

Japikse, D. (1984). Turbomachinery Diffuser Design Technology. DTS-1, Concepts ETI, Vermont.

Johnston, J.P. and Dean, R.C. (1966). Losses in vaneless diffusers of centrifugal compressors and pumps. Journal of Engineering for Power, Vol. 88, pp. 49-62.

Krain, H. (1981). A Study on Centrifugal Impeller and Diffuser Flow. Journal of Engineering for Gas Turbine and Power, Vol. 103.

Majidi, K. (2005). Numerical Study of Unsteady Flow in a Centrifugal Pump. Journal of Turbomachinery, Vol. 127, pp. 363-371.

Meakhail, T. and Park, S.O. (2005). A Study of Impeller-Diffuser-Volute Interaction in a Centrifugal Fan. Journal of Turbomachinery, Vol. 127, pp. 84-90.

Runstadler, P.W. and Dolan, F.X. (1973). Further data on the pressure recovery performance of straight channel, plane divergence diffusers at high subsonic Mach numbers. Journal of Fluids Engineering, Vol. 95, p. 373-.

Stanitz, J.D. (1952). One-dimensional compressible flow in vaneless diffusers of radial and mixed flow centrifugal compressors. Trans. of the ASME, Vol. 74, pp 473-. 